From the Department of Hematologic Oncology and Blood Disorders, Cleveland Clinic Taussig Cancer Institute, Cleveland, $\mathrm{OH} ; \mathrm{H}$. Lee Moffitt Cancer Center and Research Institute; James A. Haley Veterans Affairs Hospital and University of South Florida College Of Medicine, Tampa, FL; University of California, Los Angeles, Los Angeles, CA; and Pennsylvania State University, Hershey, PA.

Submitted September 10, 2009 ; accepted December 29, 2009; published online ahead of print at www.jco.org on March 29, 2010.

Written on behalf of the Rare Diseases Clinical Research Network Bone Marrow Failure Consortium.

Supported in part by National Institutes of Health Grant No. U54RR19397-03 (M.A.S., A.P.L., R.P., T.L., and J.P.M.).

Presented in part at the 49th Annual Meeting of the American Society of Hematology, December 8-11, 2007, Atlanta, GA; and the 50th Annual Meeting of the the American Society of Hematology, December 9-12, 2008, San Francisco, CA

Authors' disclosures of potential conflicts of interest and author contributions are found at the end of this article.

Clinical Trials repository link available on JCO.org.

Corresponding author: Mikkael A Sekeres, MD, MS, Myelodsyplastic Syndromes Program, Department of Hematologic Oncology and Blood Disorders, Cleveland Clinic Taussig Cancer Institute, Desk R35, 9500 Euclid Ave, Cleveland, $\mathrm{OH} 44195$; e-mail: sekerem@ccf.org

(C) 2010 by American Society of Clinical Oncology

0732-183X/10/2813-2253/\$20.00

DOI: $10.1200 / \mathrm{JCO} 2009.26 .0745$

\title{
Phase I Combination Trial of Lenalidomide and Azacitidine in Patients With Higher-Risk Myelodysplastic Syndromes
}

Mikkael A. Sekeres, Alan F. List, David Cuthbertson, Ronald Paquette, Rebecca Ganetsky, Deborah Latham, Katarina Paulic, Manuel Afable, Hussain I. Saba, Thomas P. Loughran Jr, and Jaroslaw P. Maciejewski

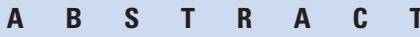

\section{Purpose}

Lenalidomide and azacitidine are active in patients with lower- and higher-risk myelodysplastic syndromes (MDS). These agents may complement each other by targeting both the bone marrow microenvironment and hypomethylating action on the malignant clone.

\section{Patients and Methods}

This phase I trial explored the safety of combination therapy in patients with higher-risk MDS. Response and characterization of molecular and methylation status of responders were secondary objectives. Patients were enrolled using a $3+3$ dose escalation. Cycles lasted 28 days, and patients received a maximum of seven cycles.

\section{Results}

Of 18 patients enrolled, median age was 68 years (range, 52 to 78 years), interval from diagnosis was 5 weeks (range, 2 to 106 weeks), and follow-up was 7 months (range, 1 to 26 months). International Prognostic Scoring System categories were intermediate $1(\mathrm{n}=2)$, intermediate 2 $(n=10)$, and high $(n=6)$. No dose-limiting toxicities occurred, and a maximum-tolerated dose was not reached. Grades 3 to 4 nonhematologic toxicities $(>1)$ included febrile neutropenia $(n=5)$, cardiac ( $n=2)$, and CNS hemorrhage $(n=2)$. Median absolute neutrophil count decrease was $26 \%$, and platelet decrease was 1\% (mean, 24\%). The overall response rate was 67\%: eight patients $(44 \%)$ had a complete response (CR); three patients $(17 \%)$ had hematologic improvement; one patient $(6 \%)$ had marrow CR. Patients achieving CR were more likely to have normal cytogenetics and lower methylation levels.

\section{Conclusion}

The combination of lenalidomide and azacitidine is well tolerated with encouraging clinical activity. The go-forward dose is azacitidine $75 \mathrm{mg} / \mathrm{m}^{2}$ on days 1 through 5 and lenalidomide $10 \mathrm{mg}$ on days 1 through 21.

\section{J Clin Oncol 28:2253-2258. (C) 2010 by American Society of Clinical Oncology}

\section{INTRODUCTION}

The myelodysplastic syndromes (MDS) comprise a spectrum of distinct bone marrow disorders associated with cytopenias, a consequential increased risk of bleeding and infection, and in higher-risk subtypes (commonly defined as patients with excess myeloblasts or an International Prognostic Scoring System [IPSS] risk score $>1.0$ ), a high likelihood of transformation to acute myeloid leukemia (AML).,

The age-adjusted annual incidence rate of MDS in the United States is 3.4 per 100,000 people, translating to greater than 10,000 new occurrences per year. ${ }^{3,4}$ Approximately $25 \%$ to $30 \%$ of recently diagnosed patients have higher-risk MDS, whereas only $15 \%$ to $20 \%$ of established patients have advanced disease. ${ }^{5}$
Three drugs, azacitidine, decitabine, and lenalidomide, were approved by the US Food and Drug Administration for the treatment of MDS, or one of its subtypes. $^{6-8}$ Though the mechanism of action of lenalidomide has not been definitively determined, it purportedly works through inhibition of phosphatase activity in the common deleted region that plays a key role in cell cycle regulation; through a defect in ribosomal protein function; via direct cytotoxic mechanisms in patients with the del $(5 q)$ cytogenetic abnormality ${ }^{9,10}$; and, supposedly, through effects on the bone marrow microenvironment in patients who do not have this lesion. ${ }^{11-14}$ Azacitidine and decitabine exert their effects via DNA methyltrasferase inhibition and also via direct cytotoxicity. ${ }^{15}$ Each drug has an impact on peripheral cytopenias, transfusion needs, and quality of life in responding patients. ${ }^{16,17}$ Additionally, azacitidine 
improves overall survival in patients with higher-risk MDS or oligoblastic acute myeloid leukemia (AML) when compared with patients treated with conventional care. ${ }^{18}$

The next frontier in MDS therapeutics is to combine active agents with different mechanisms of action, particularly in higher-risk disease, for which both microenvironment and cell regulatory mechanisms play a role. We performed a phase I study to investigate the safety, tolerance, and response rates of combination therapy with lenalidomide and azacitidine in patients with higher-risk MDS, and we explored the relationship between genomic methylation and molecular features to clinical response.

\section{PATIENTS AND METHODS}

\section{Study Design}

This was a multicenter, single-arm, open-label, phase I study of combination therapy with lenalidomide and azacitidine. The study received local institutional review board approval from all participating sites and from the Data Safety and Monitoring Board of the Rare Diseases Branch of the National Institutes of Health, and it was registered with http//:clinicaltrials.gov. The study opened in May 2005 at Cleveland Clinic and opened by December 2005 at all sites; the last patients were enrolled in May 2008. During this period, the study was placed on hold for a total of 8 months as information regarding toxicities was assessed.

The primary objective was to determine the maximum-tolerated dose (MTD) and dose-limiting toxicity (DLT) of combination therapy in patients with higher-risk MDS. A secondary objective was to report the response rate, as defined by 2006 International Working Group (IWG) criteria. ${ }^{19}$ Patients were enrolled by using a standard $3+3$, phase I design with dosing modifications of both drugs, as detailed in Table 1. Azacitidine dosing was based on preliminary data from a study of alternative azacitidine dosing schedules, which demonstrated similar efficacy in patients with MDS $^{20}$ Lenalidomide dosing was based on the syncopated schedules used in the initial phase I/II study in patients with MDS, with escalation to the single-agent therapeutic dose of 10 mg daily. ${ }^{21}$ Combination was based on presumed nonoverlapping mechanisms of action. Patients received up to seven cycles of therapy and could continue on single-agent azacitidine off study thereafter. Each cycle lasted 28 days.

\section{Patients}

A total of 25 patients were screened at all four participating institutions of the Rare Diseases Network Bone Marrow Failure Consortium and were enrolled from Cleveland Clinic and the H. Lee Moffit Cancer Center. Patients were older than 18 years of age and had MDS of one of the following FrenchAmerican-British classifications ${ }^{22}$ : refractory anemia with excess blasts (RAEB) excluding patients with exactly $20 \%$ blasts, now considered AML; or chronic myelomonocytic leukemia (CMML) with $10 \%$ to $19 \%$ myeloblasts in the bone marrow and/or 5\% to $19 \%$ blasts in the blood. Alternatively, patients

\begin{tabular}{|c|c|c|}
\hline Dose Level & Azacitidine Schedule & Lenalidomide Schedule \\
\hline 1 & $75 \mathrm{mg} / \mathrm{m}^{2} \mathrm{SC}$ days $1-5$ & 5 mg PO days 1-14 \\
\hline 2 & $75 \mathrm{mg} / \mathrm{m}^{2} \mathrm{SC}$ days $1-5$ & 5 mg PO days 1-21 \\
\hline 3 & $75 \mathrm{mg} / \mathrm{m}^{2} \mathrm{SC}$ days $1-5$ & 10 mg PO days 1-21 \\
\hline 4 & $50 \mathrm{mg} / \mathrm{m}^{2} \mathrm{SC}$ days $1-5,8-12$ & 5 mg PO days 1-14 \\
\hline 5 & $50 \mathrm{mg} / \mathrm{m}^{2} \mathrm{SC}$ days $1-5,8-12$ & 5 mg PO days 1-21 \\
\hline 6 & $50 \mathrm{mg} / \mathrm{m}^{2} \mathrm{SC}$ days $1-5,8-12$ & 10 mg PO days $1-21$ \\
\hline
\end{tabular}

NOTE. Each cycle of therapy lasted 28 days, and patients were treated for a maximum of seven cycles.

Abbreviations: SC, subcutaneously; PO, orally. could have one of the following WHO classifications: CMML-2; refractory anemia with excess blasts-1; or refractory anemia with excess blasts- $2 .{ }^{23} \mathrm{~Pa}-$ tients with RAEB in transformation were excluded, as the WHO now defines these patients as having AML, and by design the study was limited to patients with MDS, because results from the azacitidine survival study were not yet available. ${ }^{18}$ Alternatively, patients could have an IPSS score ${ }^{22,24}$ of intermediate 2 (Int-2; ie, 1.5 to 2.0 points) or high (ie, $>2.5$ points) in the setting of greater than 5\% myeloblasts. Patients had to have an Eastern Clinical Oncology Group status less than 2 and had to not be candidates for bone marrow transplantation for first-line therapy, as assessed by patients declining this treatment option. Initially, patients had to have a minimal platelet count of $50,000 / \mu \mathrm{L}$, though this was amended to $20,000 / \mu \mathrm{L}$ in fall 2006.

\section{Toxicity Assessment}

To determine the MTD, DLT was defined as greater than grade 3 nonhematologic toxicity (by using National Cancer Institute Common Toxicity Criteria [NCI CTC], version 3.0); inability to deliver the full dosing schedule of daily lenalidomide according to the assigned dose level because of toxicity; febrile neutropenia; grade 4 neutropenia in patients with absolute neutrophil count greater than 1,000 at enrollment; and inability to initiate cycle 2, day 1 of therapy within 28 days of anticipated start. Hematologic toxicities were recorded throughout the study and are reported as mean or median percentage decreases from baseline occurring during the first 8 weeks of therapy. DLTs were assessed during the first cycle of therapy within each treatment group. All patients who received any study drug were included in analyses for safety and efficacy.

\section{Efficacy Assessment}

Response was assessed by using 2006 International Working Group MDS response criteria after four cycles of therapy. ${ }^{19}$ Participants continued to receive therapy for an additional three cycles with stable disease, hematologic improvement (HI), complete response (CR), or partial response (PR). ${ }^{6}$ Response was again assessed after seven cycles of therapy. Patients were discontinued from therapy as a result of relapse occurrences after CR or PR, unacceptable toxicity, or disease progression. Responding patients could continue on single-agent azacitidine therapy off study per investigator discretion, and these patients were observed until nonresponse or disease progression, death, or study data lock (June 2009) occurred.

\section{Single Nucleotide Polymorphism Array-Based Karyotyping}

In addition to routine metaphase cytogenetics, we also performed single nucleotide polymorphism array (SNP-A) -based karyotyping to improve detection of unbalanced chromosomal aberrations by using the Affymetrix GeneChip Human Mapping 250K Array and Genome-Wide Human SNP Array 6.0 (Affymetrix, Santa Clara, CA), as previously described. ${ }^{25}$ Copy number variations and areas of uniparental disomy (UPD) were investigated using a Hidden Markov Model and CN Analyzer for Affymetrix GeneChip Mapping 250K arrays (version 3.0, Affymetrix). ${ }^{26,27}$

\section{Methylation Analysis}

The Infinium HumanMethylation27k BeadChip (Illumina, San Diego, CA) was used for methylation detection per manufacturer's protocol, as previously described. ${ }^{15}$ Methylation status of the interrogated CpG sites was calculated as a ratio of the fluorescence signals corresponding to methylated and unmethylated status ( $\beta$ value), which was compared with control values (determined by analysis of 26 bone marrow samples from healthy individuals). Aberrent hypermethylation was defined as a $\beta$ value for a $\mathrm{CpG}$ site significantly greater $(P<.001)$ than the average $\beta$ value of the same CpG site in the control group. Hypermethylated sites were defined as greater than $75 \%$ methylation of controls for each gene.

\section{Mutational Analysis}

Screening for mutations in TET2 was carried out by using direct genomic sequencing, as previously described. ${ }^{28}$ Sequencing was performed by standard techniques with the ABI 3730xl DNA analyzer (Applied Biosystems, Foster City, CA). To screen patients for mutations in c-Cbl (exon 8-9), Cbl-b (exon 9 to 10 ), and $\mathrm{Cbl-c}$ (exon 7 to 8 ), direct genomic sequencing was performed as described for the TET2 gene. ${ }^{28}$ Screening for JAK2V617F mutation was performed as previously described. ${ }^{29}$ 


\section{Statistics}

Demographic and baseline MDS disease characteristics of all patients were summarized descriptively. Severe adverse events and efficacy were summarized for the entire treated population and within dosing cohorts. The safety of the combination was evaluated by the frequency and severity of adverse events (according to NCI CTC, version 3.0) that occurred during the first 4 weeks of therapy and throughout the seven-cycle study period. The incidence and percentage of adverse events were summarized overall and within each dosing cohort.

Objective IWG responses were reported by the investigator at each participating institution and were assessed by the primary investigator of the overall study (M.A.S.). The responses are summarized for the entire cohort by using percentages. Statistical significance of the difference of methylation level between different groups was assessed using the $t$ test.

\begin{tabular}{|c|c|}
\hline Characteristic & Patients $(\mathrm{N}=18)$ \\
\hline \multicolumn{2}{|l|}{ Age, years } \\
\hline Median & 68 \\
\hline Range & $52-78$ \\
\hline \multicolumn{2}{|l|}{ Sex } \\
\hline Male & 11 \\
\hline Female & 7 \\
\hline \multicolumn{2}{|c|}{ Time from diagnosis, weeks } \\
\hline Median & 5 \\
\hline Range & $2-106$ \\
\hline \multicolumn{2}{|l|}{ Baseline $\mathrm{Hgb}, \mathrm{g} / \mathrm{dL}$} \\
\hline Median & 9.8 \\
\hline Range & $7-15$ \\
\hline \multicolumn{2}{|l|}{ Platelets, $\times 10^{3} / \mathrm{mL}$} \\
\hline Median & 69 \\
\hline Range & $19-243$ \\
\hline \multicolumn{2}{|l|}{ ANC, $\times 10^{3} / \mathrm{mL}$} \\
\hline Median & 0.84 \\
\hline Range & $0.04-13.4$ \\
\hline \multicolumn{2}{|l|}{ Epo, MIU/mL } \\
\hline Median & 95 \\
\hline Range & $19-1,316$ \\
\hline \multicolumn{2}{|l|}{ Blast \% } \\
\hline Median & 12 \\
\hline Range & $7-19$ \\
\hline \multicolumn{2}{|l|}{ IPSS } \\
\hline Int-1 & 2 \\
\hline Int-2 & 10 \\
\hline High & 6 \\
\hline \multicolumn{2}{|l|}{ WHO classification } \\
\hline CMML-2 & 1 \\
\hline RAEB-1 & 3 \\
\hline RAEB-2 & 14 \\
\hline \multicolumn{2}{|l|}{ Prior therapy } \\
\hline \multicolumn{2}{|l|}{ Growth factor } \\
\hline No. & 5 \\
\hline$\%$ & 28 \\
\hline \multicolumn{2}{|l|}{ Immunosuppressant } \\
\hline No. & 3 \\
\hline$\%$ & 17 \\
\hline \multicolumn{2}{|l|}{ Chemotherapy } \\
\hline No. & 4 \\
\hline$\%$ & 22 \\
\hline $\begin{array}{l}\text { Abbreviations: Hgb, } \\
\text { erythropoietin; IPSS, Int } \\
\text { diate } 1 \text {; Int-2, intermec } \\
\text { RAEB, refractory anemi }\end{array}$ & $\begin{array}{l}\text { rophil count; Epo, } \\
\text { tem; Int-1, interme- } \\
\text { onocytic leukemia; }\end{array}$ \\
\hline
\end{tabular}

\section{RESULTS}

\section{Patients}

A total of 18 patients with histologically confirmed MDS were enrolled across the six dosing cohorts (Table 1). Baseline characteristics appear in Table 2. Two patients had an IPSS risk classification of intermediate 1 (Int-1; both with excess blasts); 10 had Int-2; and six had high. Patients were in the following WHO classifications: CMML-2 $(\mathrm{n}=1)$; RAEB-1 $(\mathrm{n}=3)$; and RAEB-2 $(\mathrm{n}=14)$. Prior therapies included growth factors $(n=5 ; 28 \%)$, immunosuppressants $(\mathrm{n}=3 ; 17 \%)$, or cytotoxic chemotherapy (hydroxyurea or cytarabine, $\mathrm{n}=4 ; 22 \%)$.

\section{Assessment of Safety}

No DLTs were reached through all dosing cohorts. Patients received a median of five cycles of therapy (range, one to seven cycles). During the first 8 weeks of therapy, the median absolute neutrophil count decrease was $26 \%$, and the mean platelet decrease was $24 \%$ (median, 1\%). Cycle 2 was delayed in five patients for less than 9 days. Thus, no MTD was reached. Grade 3 or 4 nonhematologic toxicities that occurred at any time from cycle 2 through cycle 7 are detailed in Table 3; the most common of these were febrile neutropenia $(n=5)$, CNS hemorrhage ( $n=2)$, and cardiac $(n=2)$. Table 4 lists nonserious, nonhematologic adverse events occurring in greater than $10 \%$ of patients. Table 5 lists nonhematologic serious adverse events within each dosing cohort; the events appeared to increase with cohorts in which azacitidine was administered by using 10 -day dosing (ie, cohorts 4 to 6$)$.

\section{Assessment of Efficacy}

Patients were observed for a median of 7 months (range, 1 to 26 months). Of 18 evaluable patients, 12 (67\%) responded to therapy: eight (44\%) achieved CR, three (17\%) had hematologic improvement $(17 \%)$, and one (6\%) had bone marrow CR. Two patients (11\%) had progressive disease within 3 months of enrollment, whereas four patients $(22 \%)$ had stable disease. Initial responses occurred at a median of 3.5 months (range, 1.5 to 7 months) after the start of therapy and were sustained for a median of 8 months (range, 2 to $\geq 17$ months). Of those who responded, eight experienced relapse or experienced disease progression at a median of 7.5 months from initial response (range, 3 to 17 months); of these, two patients experienced disease

Table 3. Grade 3 or 4 Nonhematologic Toxicities of Entire Population

\begin{tabular}{lcc}
\hline \multicolumn{1}{c}{ Events } & No. of Events & Dosing Cohort of Event \\
\hline Cardiac* & 2 & 1,5 \\
Monocular blindness & 1 & 2 \\
Basal-cell skin carcinoma & 1 & 2 \\
CNS hemorrhage & 2 & 4,5 \\
Febrile neutropenia & 5 & $1,2,4,5,5$ \\
Shortness of breath & 1 & 4 \\
Perforated appendix & 1 & 6 \\
Renal failure & 1 & 5 \\
Infection & 1 & 6 \\
\hline NOTE. Toxicities may have occurred at any time between cycle 2 and cycle \\
7 of therapy. \\
*Atrial fibrillation and hypotension (n $=1$ each).
\end{tabular}




\begin{tabular}{|c|c|c|c|c|}
\hline \multirow[b]{2}{*}{ Adverse Event } & \multicolumn{2}{|c|}{$\begin{array}{l}\text { Patients } \\
\text { Affected }\end{array}$} & \multicolumn{2}{|c|}{$\begin{array}{c}\text { Events } \\
\text { Possibly } \\
\text { Related to } \\
\text { Treatment }\end{array}$} \\
\hline & No. & $\%$ & No. & $\%$ \\
\hline Constipation & 11 & 61 & 7 & 39 \\
\hline Diarrhea & 10 & 56 & 8 & 44 \\
\hline Injection site reaction & 9 & 50 & 9 & 50 \\
\hline Pruritis & 7 & 39 & 7 & 39 \\
\hline Rash/desquamation & 6 & 33 & 5 & 28 \\
\hline Fatigue & 6 & 33 & 4 & 22 \\
\hline Nausea & 5 & 28 & 5 & 28 \\
\hline Dizziness & 5 & 28 & 1 & 6 \\
\hline Fever (without neutropenia) & 4 & 22 & 2 & 11 \\
\hline Insomnia & 3 & 17 & 1 & 6 \\
\hline Dyspepsia & 3 & 17 & 3 & 17 \\
\hline Headache & 3 & 17 & 0 & 0 \\
\hline Cough & 3 & 17 & 0 & 0 \\
\hline Dyspnea & 3 & 17 & 0 & 0 \\
\hline
\end{tabular}

NOTE. Nonserious was defined as grade $\leq 2$.

*Toxicities may have occurred at any time between cycle 2 and cycle 7 of therapy.

evolution to AML, one patient at 7 and one patient at 11 months from initial response. Only one patient, in dosing cohort 4, had a del(5q) cytogenetic abnormality, and this patient achieved a CR that lasted for 7 months before AML progression occurred.

\section{Determination of Phase II Dose}

As no MTD was determined, determination of phase II dosing was made by consensus of the investigators. Toxicities increased with dosing cohorts 4 to 6 of azacitidine without appreciable increases in responses. It was the unanimous consensus that dosing cohort 3 yielded the lowest toxicity (measured by grade 3 or 4 nonhematologic toxicities) in combination with the best responses ( $\mathrm{CR}, \mathrm{n}=2$; stable disease, $\mathrm{n}=1$ ) among the first three dosing cohorts. There also did not appear to be an increase in hematologic toxicity when dosing cohort 3 was compared with cohorts 1 and 2. Finally, duration of response was highest for dosing cohort 3 , at a mean of $\geq 14$ months compared with 5.5 months for cohort 1 and 10.5 months for cohort 4 . Thus, dosing cohort 3 was selected as the phase II dose.

\section{Molecular Analysis of Chromosomal Defects With SNP-A-Based Karyotyping}

For 14 patients, both metaphase cytogenetics and SNP-A-based karyotyping were performed (Table 6). By routine cytogenetics, seven (50\%) of 14 patients showed normal karyotype; three patients had complex cytogenetics, two patients had trisomy 8 , and one patient showed del5q31.1. By SNP-A karyotyping, previously cryptic chromosomal lesions were detected in one patient with a normal metaphase karyotype. Additional defects also were detected in three patients with abnormal cytogenetics by routine testing. In particular, three areas of somatic UPD were found: UPD19p, UPD8q, and UPD11q. A CR was achieved in six of seven patients with normal chromosomes but only in the patient with $\operatorname{del}(5 \mathrm{q} 31.1)$, of seven patients with an abnormal karyotype.

\section{Methylation Array Assessment}

When analyzed as a group, increased methylation levels and numbers of methylated sites were found in all patients compared with controls before treatment (on average, 620 differentially hypermethylated genes; ie, mean $\beta$ value 0.358 in patients $v 0.337$ in controls). After treatment, both methylation levels and the number of methylated promoters decreased in patients achieving CR $(P=.02$ and $P<.001$, respectively), whereas no differences were seen in patients without CR. When patients were analyzed individually, there was no correlation between pretreatment average methylation or the number of hypermethylated sites and response (Appendix Fig A1, online only).

\section{Mutational Assessment}

Patients enrolled on the trials were screened for the presence of $c$ - $C b l$ and $b$ - $C b l$ mutations; no patients, including the patient with UPD11q24, showed $c$ - $C b l$ mutations. All patients were also negative for the JAK2V617F mutation. TET2 mutations were identified in two patients: one had a normal karyotype, whereas trisomy 8 was found in

\begin{tabular}{|c|c|c|c|c|}
\hline AZA Dose by Dosing Cohort & LEN Dose & IPSS Risk Group & $\begin{array}{c}\text { Grade } 3 \text { to } 4 \\
\text { Nonhematologic Toxicities* }\end{array}$ & Maximum Response \\
\hline \multicolumn{5}{|l|}{1} \\
\hline $75 \mathrm{mg} / \mathrm{m} 2 \mathrm{SC}$ days $1-5$ & 5 mg PO days $1-14$ & 1 Int-1, 2 Int-2 & 2 & $2 \mathrm{CR}, 1$ progression \\
\hline \multicolumn{5}{|l|}{2} \\
\hline $75 \mathrm{mg} / \mathrm{m} 2$ SC days $1-5$ & 5 mg PO days $1-21$ & 2 Int-2, 1 high & 2 & $1 \mathrm{CR}, 1 \mathrm{HI}, 1$ stable disease \\
\hline \multicolumn{5}{|l|}{3} \\
\hline $75 \mathrm{mg} / \mathrm{m} 2 \mathrm{SC}$ days $1-5$ & $10 \mathrm{mg} P O$ days $1-21$ & 1 Int-2, 2 high & 0 & $2 \mathrm{CR}, 1$ stable disease \\
\hline \multicolumn{5}{|l|}{4} \\
\hline 50 mg/m2 SC days 1-5, 8-12 & $5 \mathrm{mg} P \mathrm{PO}$ days $1-14$ & 1 Int-1, 2 Int-2 & 2 & 2 CR, 1 stable disease \\
\hline \multicolumn{5}{|l|}{5} \\
\hline $50 \mathrm{mg} / \mathrm{m} 2 \mathrm{SC}$ days $1-5,8-12$ & $5 \mathrm{mg} P O$ days $1-21$ & 2 Int-2, 1 high & 5 & $1 \mathrm{HI}, 1$ stable disease, 1 progression \\
\hline \multicolumn{5}{|l|}{6} \\
\hline 50 mg/m2 SC days 1-5, 8-12 & 10 mg PO days $1-21$ & 1 Int-1, 1 Int-2, 1 high & 2 & $1 \mathrm{CR}, 1 \mathrm{HI}, 1 \mathrm{BM} C \mathrm{CR}$ \\
\hline
\end{tabular}




\begin{tabular}{|c|c|c|c|c|}
\hline Patient & Cytogenetics* & $\begin{array}{c}c-C b / / b-C b / / \\
\text { JAK2 }\end{array}$ & TET2 & Response \\
\hline 1 & $\begin{array}{l}47, x y,+8[20] \text { UPD19p13- } \\
11 \text { p12 }\end{array}$ & Wt & Wt & NR \\
\hline 2 & $46, x y[20]$ & Wt & Wt & $\mathrm{CR}$ \\
\hline 3 & $46, x y[20]$ & Wt & Wt & $C R$ \\
\hline 4 & $46, x x[20] 46, x, i \times p 10[2]+$ & Wt & Wt & $C R$ \\
\hline 5 & $46, x y[20]$ & Wt & Wt & $\mathrm{CR}$ \\
\hline 6 & $46, x y[20]$ & Wt & Wt & $\mathrm{CR}$ \\
\hline 7 & $\begin{array}{l}\text { 46, xy del(5q31q35)[2] 46, } \\
\text { xy,[18] UPD6P25-3P22.1, } \\
\text { UPD8q24.3 }\end{array}$ & Wt & Wt & CR \\
\hline 8 & $46, x y[20]$ & Wt & $\begin{array}{c}\text { Heterozygous } \\
\text { T1078P }\end{array}$ & $\mathrm{CR}$ \\
\hline 9 & Complex, UPD11q24.2-q25 & Wt & Wt & NR \\
\hline 10 & $46, x \times[20]$ & Wt & $\mathrm{Wt}_{\mathrm{t}}$ & $\mathrm{HI}$ \\
\hline 11 & $46, x y[20]$ & Wt & Wt & $\mathrm{HI}$ \\
\hline 12 & Complex $\ddagger$ & Wt & ND & $\mathrm{HI}$ \\
\hline 13 & Complex $\neq$ & Wt & Wt & NR \\
\hline 14 & $47, x x,+8[20]$ & Wt & $\begin{array}{l}\text { Heterozygous } \\
\text { V1417F }\end{array}$ & NR \\
\hline \multicolumn{5}{|c|}{$\begin{array}{l}\text { Abbreviations: UPD, uniparental disomy; Wt, wild type; NR, no response; CR, } \\
\text { complete response; HI, hematologic improvement; ND, sufficient DNA not } \\
\text { available. } \\
\text { "Metaphase cytogenetics + SNP-A karyotyping (with } 6.0 \text { Affymetrix, Santa } \\
\text { Clara, CA). } \\
\text { +Not considered abnormal. } \\
\text { FIncluding del(7) and del(5). }\end{array}$} \\
\hline
\end{tabular}

the other. TET2 mutations may explain more aggressive features of MDS. One of these patients achieved a response.

\section{DISCUSSION}

This is the first study to combine two drugs approved by the US Food and Drug Administration, lenalidomide and azacitidine, for the treatment of MDS. The justification for this strategy rested in the theory that patients with higher-risk MDS, in which pro-proliferative mechanisms of disease potentiation predominate, also experienced disease that retained properties of the lower-risk MDS from which the disease likely evolved. Because of this, bone marrow microenvironment and pro-inflammatory cytokine mechanisms would play a major role. Thus, the use of two agents with complementary mechanisms of action may yield additive benefit.

A concern in combining these drugs was the risk of profound myelosuppression, particularly given rates of myelosuppression that crested 50\% in registration trials of these drugs used as single agents. We found the combination to be safe in this phase I study, with no DLTs and no MTD across the six dosing cohorts. Myelosuppression was limited, which may be explained in part by how we assessed myelosuppression, with functional definitions rather than absolute NCI CTC criteria, so as to not unfairly attribute treatment-related myelosuppression to a patient entering the study with baseline severe cytopenias; and by limiting assessment to the first 8 weeks of therapy, so as not to confound the assessment with cytopenias attributable to disease progression. ${ }^{30}$ Other serious adverse events, including hemorrhage and neutropenic fever, appeared to occur with the frequency expected in a population of older patients with advanced hematologic cancer. The incidence of adverse events appeared to increase in dosing cohorts exploring the 10-day azacitidine schedule; thus, the first three dosing cohorts, in which patients received the 5-day azacitidine schedule, were judged to be more tolerable.

The combination of lenalidomide and azacitidine appeared to be at least as effective as each agent used alone. In the phase III AZA-001 European study, patients randomly assigned to receive azacitidine were a similar age (ie, 69 years) and had similar IPSS scores to this study, and they had a CR plus PR rate of $29 \% .^{18}$ Additionally, $49 \%$ of patients experienced an HI (which could include CRs and PRs). In study of lenalidomide in lower-risk patients without $\operatorname{del}(5 \mathrm{q})$, the $\mathrm{HI}$ rate was $43 \% .{ }^{31}$ In another lenalidomide study in patients with higherrisk del (5q) MDS, the CR rate was $21 \%$, and the $\mathrm{HI}$ rate was $14 \% .{ }^{32}$ In our study, the CR rate was higher than any of the other studies, at $44 \%$, and an additional $23 \%$ of patients achieved an $\mathrm{HI}$, for an overall response rate of $67 \%$. It remains to be seen whether the superior CR rate seen here is a result of patient selection (with the average patient being newly diagnosed), whether it represents a true additive or synergistic effect of combination therapy, or whether the two agents are no better than one or the other in isolation. As the cohort 3 dosing schedule resulted in two patients achieving a CR without any serious nonhematologic adverse events, this dosing schedule was chosen for phase II testing.

We also performed a comprehensive cytogenetic and molecular analysis of all patients, and we found higher CR rates among patients who were triple negatives, that is, those who have normal karyotypes by metaphase cytogenetics, SNP arrays, and additional molecular testing. Although intriguing, these results remain to be validated in larger treated cohorts because of the heterogeneity of the population and small sample size. Also, similar to other studies with DNA methyltransferase inhibitors, normal methylation patterns were restored in patients achieving a complete remission, as has been shown in patients with higher-risk compared with lower-risk MDS or compared with controls, ${ }^{15}$ though patients exhibited global hypermethylation pretreatment; however, this could not be demonstrated on an individual patient level. ${ }^{33}$ This may be due to restoration of normal hematopoiesis and underpowering of individual, as opposed to group, intrapatient methylation assessments. No DLT or MTD was identified despite the phase I nature of this study. At the time of study design, significant enough concern existed regarding additive toxicities that it was not anticipated that all six dosing cohorts would be filled and the study completed. Although higher doses of DNA methyltransferase inhibitors may not yield greater efficacy, as has been demonstrated by Saunthararajah et al, ${ }^{34}$ it would be reasonable to design a follow-up dose escalating study to explore additional dosing cohorts.

The potential benefit of combination regimens in higher-risk MDS is now entering phase II investigations. Questions that remain include duration of combination therapy; whether this regimen would be more tolerable with better efficacy if administered sequentially; and whether it would be more ideally suited for patients with higher-risk MDS with the del(5q) cytogenetic abnormality. The ultimate demonstration of an additive or synergistic effect of combination therapy will necessitate a phase III study comparing azacitidine and lenalidomide with azacitidine alone.

In conclusion, the combination of lenalidomide and azacitidine is well tolerated, with acceptable hematologic toxicities and a response rate that at least equals either agent used alone in similar populations. The phase II dose of azacitidine given at $75 \mathrm{mg} / \mathrm{m}^{2}$ daily for 5 days and lenalidomide at $10 \mathrm{mg}$ daily for 21 days was established. 
AUTHORS' DISCLOSURES OF POTENTIAL CONFLICTS OF INTEREST

Although all authors completed the disclosure declaration, the following author(s) indicated a financial or other interest that is relevant to the subject matter under consideration in this article. Certain relationships marked with a " $U$ " are those for which no compensation was received; those relationships marked with a "C" were compensated. For a detailed description of the disclosure categories, or for more information about ASCO's conflict of interest policy, please refer to the Author Disclosure Declaration and the Disclosures of Potential Conflicts of Interest section in Information for Contributors.

Employment or Leadership Position: None Consultant or Advisory Role: Mikkael A. Sekeres, Celgene (C); Alan F. List, Celgene (C) Stock Ownership: None Honoraria: Mikkael A. Sekeres, Celgene Research Funding: Mikkael A. Sekeres, Celgene; Alan F. List, Celgene; Jaroslaw P. Maciejewski, Celgene Expert Testimony: None Other Remuneration: None

\section{AUTHOR CONTRIBUTIONS}

Conception and design: Mikkael A. Sekeres, Alan F. List,

Jaroslaw P. Maciejewski

Administrative support: Deborah Latham, Katarina Paulic

Provision of study materials or patients: Mikkael A. Sekeres, Alan F.

List, Ronald Paquette, Hussain I. Saba, Thomas P. Loughran Jr,

Jaroslaw P. Maciejewski

Collection and assembly of data: Mikkael A. Sekeres, Alan F. List, David

Cuthbertson, Rebecca Ganetsky, Deborah Latham, Katarina Paulic,

Manuel Afable, Jaroslaw P. Maciejewski

Data analysis and interpretation: Mikkael A. Sekeres, David

Cuthbertson, Rebecca Ganetsky, Jaroslaw P. Maciejewski

Manuscript writing: Mikkael A. Sekeres, Jaroslaw P. Maciejewski

Final approval of manuscript: Mikkael A. Sekeres, Alan F. List, David

Cuthbertson, Ronald Paquette, Rebecca Ganetsky, Deborah Latham,

Katarina Paulic, Manuel Afable, Hussain I. Saba, Thomas P. Loughran Jr, Jaroslaw P. Maciejewski

\section{REFERENCES}

1. List AF, Vardiman J, Issa JP, DeWitte TM: Myelodysplastic syndromes. Hematology (Am Soc Hematol Educ Program) 297-317, 2004

2. Harris NL, Jaffe ES, Diebold J, et al: The World Health Organization classification of neoplastic diseases of the hematopoietic and lymphoid tissues: Report of the Clinical Advisory Committee meeting, Airlie House, Virginia, November, 1997. Ann Oncol 10:1419-1432, 1999

3. Ma X, Does M, Raza A, et al: Myelodysplastic syndromes: Incidence and survival in the United States. Cancer 109:1536-1542, 2007

4. Rollison $D E$, Hayat $M$, Smith $M$, et al: First report of national estimates of the incidence of myelodysplastic syndromes and chronic myeloproliferative disorders from the US SEER Program. ASH Annual Meeting Abstracts 108, 2006 (abstr 247)

5. Sekeres MA, Schoonen WM, Kantarjian $\mathrm{H}$, et al: Characteristics of US patients with myelodysplastic syndromes: Results of six cross-sectional physician surveys. J Natl Cancer Inst 100:1542-1551, 2008

6. Silverman $L R$, Demakos EP, Peterson $B L$, et al: Randomized controlled trial of azacitidine in patients with the myelodysplastic syndrome: A study of the cancer and leukemia group B. J Clin Oncol 20:2429-2440, 2002

7. Kantarjian $\mathrm{H}$, Issa JP, Rosenfeld CS, et al: Decitabine improves patient outcomes in myelodysplastic syndromes: Results of a phase III randomized study. Cancer 106:1794-1803, 2006

8. List A, Dewald G, Bennett J, et al: Lenalidomide in the myelodysplastic syndrome with chromosome 5q deletion. N Engl J Med 355:1456-1465, 2006

9. Wei S, Chen X, Rocha K, et al: A critical role for phosphatase haplodeficiency in the selective suppression of deletion 5q MDS by lenalidomide. Proc Natl Acad Sci U S A 106:12974-12979, 2009

10. Ebert BL, Pretz J, Bosco J, et al: Identification of RPS14 as a 5q- syndrome gene by RNA interference screen. Nature 451:335-339, 2008

11. Muller GW, Chen R, Huang SY, et al: Aminosubstituted thalidomide analogs: Potent inhibitors of TNF-alpha production. Bioorg Med Chem Lett 9:16251630, 1999
12. Dredge $K$, Marriott JB, Macdonald $C D$, et al: Novel thalidomide analogues display anti-angiogenic activity independently of immunomodulatory effects. Br J Cancer 87:1166-1172, 2002

13. Buesche G, Dieck S, Giagounidis A, et al: Antiangiogenic in vivo effect of lenalidomide (CC-5013) in myelodysplastic syndrome with del(5q) chromosome abnormality and its relation to the course of disease. Blood 106, 2005 (abstr 113)

14. Davies FE, Raje N, Hideshima T, et al: Thalidomide and immunomodulatory derivatives augment natural killer cell cytotoxicity in multiple myeloma. Blood 98:210-216, 2001

15. Jiang Y, Dunbar A, Gondek LP, et al: Aberrant DNA methylation is a dominant mechanism in MDS progression to AML. Blood 113:1315-1325, 2009

16. Balducci $L$ : Transfusion independence in patients with myelodysplastic syndromes: Impact on outcomes and quality of life. Cancer 106:2087-2094, 2006

17. Sekeres MA, Fu AZ, Maciejewski JP, et al: A decision analysis to determine the appropriate treatment for low-risk myelodysplastic syndromes. Cancer 109:1125-1132, 2007

18. Fenaux P, Mufti GJ, Hellstrom-Lindberg E, et al: Efficacy of azacitidine compared with that of conventional care regimens in the treatment of higher-risk myelodysplastic syndromes: A randomised, openlabel, phase III study. Lancet Oncol 10:223-232, 2009

19. Cheson BD, Greenberg PL, Bennett JM, et al: Clinical application and proposal for modification of the International Working Group (IWG) response criteria in myelodysplasia. Blood 108:419-425, 2006

20. Lyons RM, Cosgriff TM, Modi SS, et al: Hematologic response to three alternative dosing schedules of azacitidine in patients with myelodysplastic syndromes. J Clin Oncol 27:1850-1856, 2009

21. List $A$, Kurtin $S$, Roe $D$, et al: Efficacy of lenalidomide in myelodysplastic syndromes. N Engl J Med 352:549-557, 2005

22. Bennett JM, Catovsky D, Daniel MT, et al: Proposals for the classification of the myelodysplastic syndromes. Br J Haematol 51:189-199, 1982

23. Vardiman JW, Harris NL, Brunning RD: The World Health Organization (WHO) classification of the myeloid neoplasms. Blood 100:2292-2302, 2002

24. Greenberg P, Cox C, LeBeau MM, et al: International scoring system for evaluating prognosis in myelodysplastic syndromes. Blood 89:2079-2088, 1997
25. Gondek LP, Tiu R, O'Keefe CL, et al: Chromosomal lesions and uniparental disomy detected by SNP arrays in MDS, MDS/MPD, and MDS-derived AML. Blood 111:1534-1542, 2008

26. Flotho $C$, Kratz $C P$, Bergstrasser $E$, et al: Genotype-phenotype correlation in cases of juvenile myelomonocytic leukemia with clonal RAS mutations. Blood 111:966-967, 2008

27. Dunbar AJ, Gondek LP, O'Keefe $C L$, et al: 250K single nucleotide polymorphism array karyotyping identifies acquired uniparental disomy and homozygous mutations, including novel missense substitutions of c-Cbl, in myeloid malignancies. Cancer Res 68:10349-10357, 2008

28. Jankowska AM, Szpurka H, Tiu RV, et al: Loss of heterozygosity $4 \mathrm{q} 24$ and TET2 mutations associated with myelodysplastic/myeloproliferative neoplasms. Blood 113:6403-6410, 2009

29. Levine RL, Wadleigh M, Cools J, et al: Activating mutation in the tyrosine kinase JAK2 in polycythemia vera, essential thrombocythemia, and myeloid metaplasia with myelofibrosis. Cancer Cell 7:387-397, 2005

30. Sekeres MA, Maciejewski JP, Giagounidis $\mathrm{AA}$, et al: Relationship of treatment-related cytopenias and response to lenalidomide in patients with lower-risk myelodysplastic syndromes. J Clin Oncol 26:5943-5949, 2008

31. Raza A, Reeves JA, Feldman EJ, et al: Phase 2 study of lenalidomide in transfusion-dependent, low-risk, and intermediate-1 risk myelodysplastic syndromes with karyotypes other than deletion $5 \mathrm{q}$. Blood 111:86-93, 2008

32. Ades L, Boehrer S, Prebet T, et al: Efficacy and safety of lenalidomide in intermediate-2-or high risk myelodysplastic syndromes (MDS) with $5 q \mathrm{de}$ letion: Results of a phase II study. Blood XXXX: XXXX, 2008 (abstr XXXX)

33. Issa JP, Garcia-Manero G, Giles FJ, et al: Phase 1 study of low-dose prolonged exposure schedules of the hypomethylating agent 5-aza-2'deoxycytidine (decitabine) in hematopoietic malignancies. Blood 103:1635-1640, 2004

34. Negrotto S, Hu Z, Link KA, et al: Differentiationchronology specific function of DNMT1 and selective anti-leukemia stem-cell therapy. ASH Annual Meeting Abstracts 112:201, 2008 (abstr XXXX) 